\title{
A New Case of Hopf's Acrokeratosis Verruciformis: Clinical and Dermoscopic Features
}

\author{
Aouali $S^{1 *}$, Bouabdella $S^{1}$, Zizi $\mathbf{N}^{1,2}$ and Dikhaye $S^{1,2}$ \\ ${ }^{1}$ Department of Dermatology, Mohamed VI University Hospital, Morocco \\ ${ }^{2}$ Laboratory of Epidemiology, Mohamed the first university of Oujda, Morocco
}

*Corresponding author: Aouali Soraya, Department of dermatology, Mohamed 6 University Hospital of Oujda, Alqods, Oujda, Morocco, Tel: +212677481276; Email: soraya.aouali@gmail. com

\section{Case Report}

Volume 6 Issue 1

Received Date: January 04, 2021

Published Date: January 25, 2021

DOI: $10.23880 /$ cdoaj- 16000227

\section{Abstract}

Acrokeratosis verruciformis of Hopf (AVH) is a rare genodermatosis that has autosomal dominant inheritance. We report a new case of AVH to highlight the clinical and dermoscopic features of this rare disease.

Keywords: Acrokeratosis verruciformis of Hopf; Genodermatosis; Dermoscopy

\section{Introduction}

Acrokeratosis verruciformis of $\mathrm{Hopf}(\mathrm{AVH})$ is a rare genodermatosis characterized by keratotic lesions on the dorsum of the hands and feet. It has autosomal dominant inheritance. Most commonly, It develops during early childhood, but occasionally develops as late as the fifth decade.

We report a patient with impressive clinical and dermoscopic features.

\section{Case Report}

A 25 year-old man with Fitzpatrick skin type IV presented with multiple skin colored to brownish verrucous papules over the dorsum of his feet and distal lower limbs, and flat-topped keratotic papules and plaques over his hands, evolving for 10 years prior to his consultation.

Dermoscopic examination of feet lesions showed homogenous white areas with a cobblestone appearance. While palmar lesions presented as lobular structures (Frog spawn like) interrupting normal dermatoglyphics. Biopsy of the lesions revealed: orthokeratotic hyperkeratosis, marked acanthosis associated to papillomatosis.

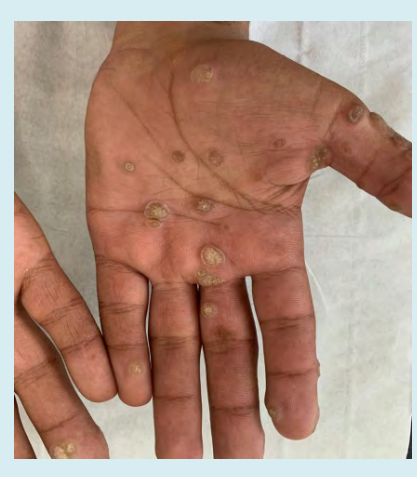

Figure 1: Keratotic palmar papules.

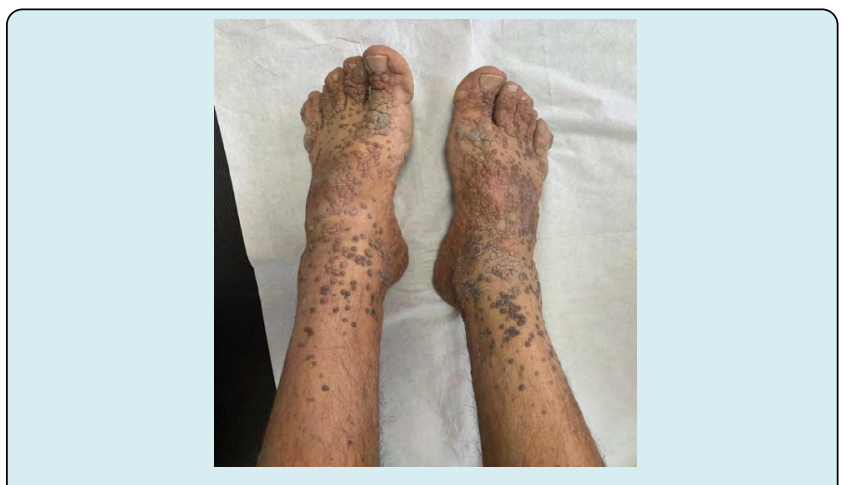

Figure 2: Multiple skin colored and brownish papules. 


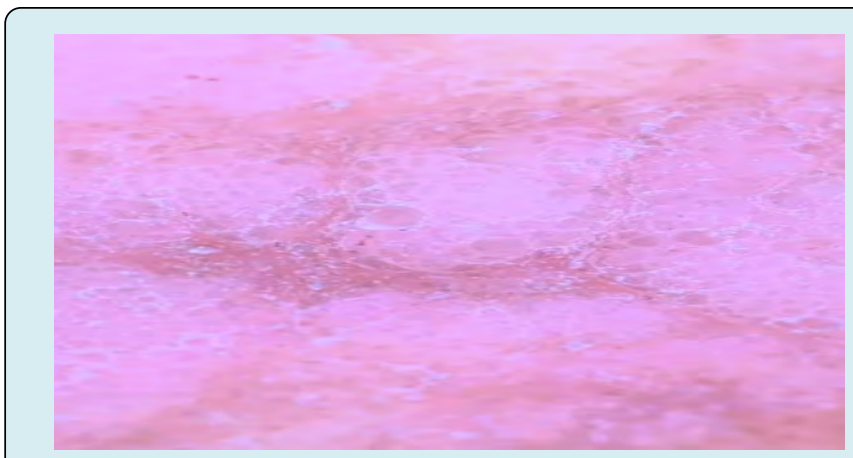

Figure 3: Dermoscopic feature: Cobblestone appearance.

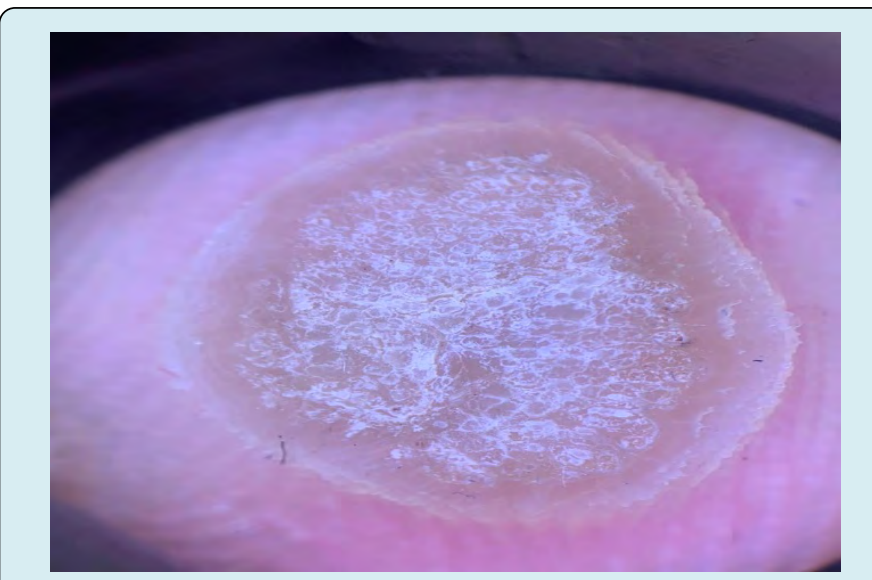

Figure 4: Dermoscopic appearance: "Frog Spawn like" pattern.

\section{Discussion}

AVH is a rare condition, with an estimated incidence of about 1 case for every 50000 visits to the dermatologist [1]. It is an autosomal dominant disorder with variable expressivity, but sporadic cases also exist. The disease has no gender preference. Its probable etiology would be a mutation in ATP2A2 gene located on chromosome 12q24 [2].

Clinically, lesions resemble flat warts and tend to develop on the dorsum of hands and feet, the toes, more rarely on the forearms, legs, elbows and knees. Punctiform hyperkeratotic lesions on the palms may also be found. The nails may also be affected [3]. Squamous cell carcinoma developped in preexisting lesions of AVH was rarely reported [4].

Recent studies described dermoscopic features of
AVH lesions, the most reported aspects were irregular white homogenous areas, white network, and cobblestone appearance [5]. Diagnosis is defined by histopathological features that include papillomatosis (circumscribed epidermal elevations known as "church spires), acanthosis, hyperkeratosis, and hypergranulosis without parakeratosis [6].

Treatment is not mandatory. No treatments have been explored in clinical trials. All the usual therapies for warts have been proposed, including freezing with liquid nitrogen, superficial excision, curettage, $\mathrm{CO} 2$ or ND: YAG laser and also local retinoid and acitretin.

\section{Conclusion}

We report a new case of AVH with specific clinical and dermoscopic features in order to facilitate the diagnosis of this rare disease.

\section{References}

1. Panja R (1977) Acrokeratosis verruciformis: (Hopf)--A clinical entity? Br J Dermatol 96(6): 643-652.

2. Bang CH, Kim HS, Park YM, Kim HO, Lee JY (2011) Non-familial acrokeratosis verruciformis of Hopf. Ann Dermatol 23: 61-63.

3. Rallis E, Economidi A, Papadakis P, Verros C (2005) Acrokeratosis verruciformis of Hopf (Hopf disease): case report and review of the litterature. Dermatol Online J 11(2): 10.

4. Farro P, Zalaudek I, Ferrara G, Fulgione E, Cicale L, et al. (2004) Unusual association between acrokeratosis verruciformis of Hopf and multiple keratoacanthomas. Successful therapy with acitretin. J Dtsch Dermatol Ges 2(6): 440-442.

5. Behera B, Prabhakaran N, Naveed S, Kumari R, Gochhait D, et al. (2017) Dermoscopy of acrokeratosis verruciformis of Hopf. J Am Acad Dermatol 77(2): 33-35.

6. Dhitavat J, Macfarlane S, Dode L, Leslie N, Sakuntabhai A, et al. (2003) Acrokeratosis verruciformis of Hopf is caused by mutation in ATP2A2: evidence that it is allelic to Darier's disease. J Invest Dermatol 120(2): 229-232. 